\title{
TSPAN 12 is overexpressed in NSCLC via p53 inhibition and promotes NSCLC cell growth in vitro and in vivo
}

This article was published in the following Dove Press journal:

OncoTargets and Therapy

\section{Zhongwu $\mathrm{Hu}^{\prime}$ \\ Daorong $\mathrm{Hou}^{2}$ \\ Xiaowei Wang ${ }^{3}$ \\ Zhenbing You' \\ Xiufeng $\mathrm{Cao}^{4}$}

'Department of Thoracic Surgery, Huai'an First People's Hospital, Nanjing Medical University, Huai'an, Jiangsu, China; ${ }^{2}$ Key Laboratory of Model Animal Research, Animal Core Facility of Nanjing Medical University, Nanjing, China; ${ }^{3}$ Department of Medical Oncology, Huai'an First People's Hospital, Nanjing Medical University, Huai'an, China; ${ }^{4}$ Department of Surgical Oncology, Nanjing First Hospital, Nanjing Medical University, Nanjing, China
Correspondence: Xiufeng Cao Department of Surgical Oncology, Nanjing First Hospital, Nanjing Medical University, 68 Changle Road, Nanjing, Jiangsu 210006, China Emailcxf55II0I@yeah.net
Background: Tetraspanin 12 (TSPAN12), a member of the phylogenetically ancient tetraspanin family, is linked to impaired vascularization of the eye called familial exudative vitreoretinopathy, while the functional role of TSPAN12 in lung cancer has not been well characterized.

Results: In our study, TSPAN12 is able to regulate the growth of non-small-cell lung carcinoma (NSCLC) cells both in vitro and in vivo. TSPAN12 mRNA level was significantly increased in human NSCLC samples compared with their corresponding paracancerous histologic normal tissues. In addition, TSPAN12 expression, which is frequently upregulated in NSCLC, is inversely correlated with p53 expression. Furthermore, the expression levels of TSPAN12 were also increased in three human NSCLC cell lines compared to human bronchial epithelial (16HBE) cells. Then, we studied the effects of TSPAN12 silencing by short hairpin ribonucleic acid on NSCLC cell growth in vitro and tumorigenesis in vivo, along with the effect on the p53 pathway. Knockdown of TSPAN12 in NSCLC cells inhibited cell proliferation and colony formation. In addition, knockdown of TSPAN12 increased apoptosis in NSCLC cells. Mechanistically, TSPAN12 could modulate the expression of p53, p21, and p27 in NSCLC cells. In a tumor xenograft model, TSPAN12 silencing inhibits the tumor growth of H1299 cells.

Conclusion: Taken together, our results reveal that TSPAN12 plays an important role in NSCLC and is a potential biomarker and a promising target in the treatment of NSCLC.

Keywords: NSCLC, TSPAN12, p53, survival, cell proliferation, apoptosis

\section{Introduction}

Non-small-cell lung carcinoma (NSCLC) is one of the leading causes of cancer-related mortality worldwide and accounts for about $85 \%$ of all lung cancers. ${ }^{1,2}$ The most common types of NSCLC are squamous cell carcinoma, large cell carcinoma, and adenocarcinoma, but there are several other types that occur less frequently. ${ }^{2}$ Aberrant expressions of proto-oncogenes and tumor-suppressive genes are involved in the carcinogenesis and development of NSCLC, which lead to tumor cell growth and tumor metastasis. ${ }^{3-6}$ Compared to small-cell lung carcinoma, NSCLC is relatively insensitive to chemotherapy. Presently, chemotherapy is increasingly being used to treat NSCLC both preoperatively (neoadjuvant chemotherapy) and postoperatively (adjuvant chemotherapy), and surgical resection is still the primary therapy, but the 5 -year overall survival rate is just $16 \%$ for all stages. ${ }^{7}$ Considering the complex nature of NSCLC, it is of great importance to discover molecular mechanisms involved in initiation and progression of NSCLC.

Tetraspanin 12 (TSPAN12) is a member of tetraspanin family, characterized by four transmembrane domains and two extracellular loops. ${ }^{8}$ Some of tetraspanin family 
proteins are shown to be involved in the suppression of metastasis (eg, TSPAN27 and TSPAN29) and tumor progression (eg, TSPAN8 and TSPAN24). ${ }^{9-12}$ Most studies on TSPAN12 have been performed on familial exudative vitreoretinopathy, an inherited blinding disorder of the retinal vascular system. ${ }^{13,14}$ In vivo functional analyses using TSPAN12-deficient mice revealed that TSAPN12 contributed to retinal vascular development by cooperating with FZD4 and LRP5, and regulated Norrin-induced $\beta$-catenin signaling. ${ }^{14}$ Furthermore, TSPAN12 has been shown to promote the maturation of tumor-facilitating sheddase ADAM10 and supports human breast cancer growth. ${ }^{15,16}$ TSPAN12 knockdown in p53-depleted fibroblasts inhibited cancer cell proliferation and invasion in vitro, and inhibited tumor growth in vivo. ${ }^{17}$ Thus, TSPAN12 has been suggested to play a role in cancer progression.

Approximately half of the human cancers result from p53 inactivation by mutations and deletations. ${ }^{17}$ p53 is known as a transcription factor that regulates the expression of genes associated with cell-cycle arrest, apoptosis, and senescence to prevent tumorigenesis. ${ }^{18,19}$ The tumor suppressor $\mathrm{p} 53$ plays a key role in cancer biology and represents an attractive target for the development of anticancer therapies. ${ }^{20}$

\section{Materials and methods Patients and samples}

This study was approved by the Ethics Committee of Huai'an First People's Hospital, Nanjing Medical University, Huai'an, Jiangsu, China. Paracarcinoma lung tissues and NSCLC tissues $(n=16)$ were collected from NSCLC patients enrolled in Huai' an First People's Hospital, Nanjing Medical University. Written informed consent was obtained from all patients and patients underwent curative surgery without prior treatments. Tissue samples were snap-frozen in liquid nitrogen and stored at $-80^{\circ} \mathrm{C}$ until analysis.

\section{Immunohistochemistry $(\mathrm{IHC})$ analysis}

IHC was performed to detect the TSPAN12 expression level in NSCLC tissues and normal adjacent tissues by the avidin-biotin-peroxidase complex method. All sections were deparaffinized in xylene and dehydrated through a gradient concentration of alcohol. Then endogenous peroxidase activity was blocked using $0.5 \% \mathrm{H}_{2} \mathrm{O}_{2}$ in methanol for $10 \mathrm{~min}$. After nonspecific binding was blocked, the slides were incubated with rabbit polyclonal antibodies for TSPAN12 (1:100) in $1 \times \mathrm{PBS}$ at $4^{\circ} \mathrm{C}$ overnight in a humidified container. Next day, biotinylated goat anti-rabbit immunoglobulin $\mathrm{G}$ (IgG) (1:400; Sigma-Aldrich Co.) was incubated with the sections for $1 \mathrm{~h}$ at room temperature and detected using a streptavidin peroxidase complex. The brown color indicative of peroxidase activity was developed by incubation with $0.1 \%$ 3, 3'-diaminobenzidine (Sigma-Aldrich Co.) in $1 \times \mathrm{PBS}$ with $0.05 \% \mathrm{H}_{2} \mathrm{O}_{2}$ for $5 \mathrm{~min}$ at room temperature.

\section{Cell lines}

Human lung carcinoma cell lines (A549, H1299, H1650) were purchased from the Cell Bank of the Chinese Academy of Sciences (Shanghai, China), and human bronchial epithelial (16HBE) cells were from Bogoo Biotechnology (Shanghai, China). These cells were seeded and cultured in RPMI 1640 medium (HyClone) with 10\% fetal bovine serum (FBS; Thermo Fisher Scientific). HEK293T cells were obtained from the Institute of Cytobiology, Chinese Academy of Sciences, and maintained in Dulbecco's Modified Eagle's Medium (DMEM; Thermo Fisher Scientific) with 10\% FBS. All cells were cultured in a $37^{\circ} \mathrm{C}$ cell incubator with $5 \% \mathrm{CO}_{2}$. The authentication of all cell lines had been confirmed.

\section{Lentivirus transduction and infection}

For lentivirus packaging, HEK293T cells $\left(7 \times 10^{6}\right)$ were plated in a $15 \mathrm{~cm}$ dish with DMEM containing $10 \%$ FBS for $24 \mathrm{~h}$, and then transfected with $15 \mu \mathrm{g}$ of lentivirus plasmids. The virus containing medium was harvested after transfection for 48 and $72 \mathrm{~h}$. The virus was filtered through a $0.45 \mu \mathrm{m}$ filter (EMD Millipore) and centrifuged at $20,000 \times g$ for $2 \mathrm{~h}$. The precipitate was suspended in $100 \mu \mathrm{L}$ DMEM. For infections, gradient amount of lentivirus was added into the culture medium of H1299 cells with polybrene ( $4 \mu \mathrm{g} / \mathrm{mL}$; SigmaAldrich, Co.). After incubation at $37^{\circ} \mathrm{C}$ for $36-48 \mathrm{~h}$, the infected cell populations were confirmed by fluorescence microscope for green fluorescent protein expression to evaluate the virus titer.

\section{RNA extraction, cDNA synthesis, and qRT-PCR}

Cells and tissues were lysed by Trizol reagent (Thermo Fisher Scientific). Total RNA was purified and reverse transcribed to cDNA using avian myeloblastosis virus reverse transcriptase (Takara) according to the manufacturer's instructions. The quantification of target gene transcripts was performed using SYBR Green Realtime PCR Master Mix (Toyobo) on an ABI Prism 7900 sequence detection system (Applied Biosystems). The primer sequences for quantitative reverse transcriptase polymerase chain reaction (qRT-PCR) of TSPAN12 and $\beta$-actin were 
as follows: 5'-TGGCCAGAGAAGATTCCGTGA-3' and 5'-GACTGCTTCCTCTACCCTCGTT' for TSPAN12, 5'-GATCATTGCTCCTCCTGAGC-3' and 5'-ACTCCTGCTTGCTGATCCAC-3' for $\beta$-actin. mRNA expression values of TSPAN12 were normalized to the internal control $\beta$-actin. Relative expression was calculated using the cycle threshold $(\mathrm{Ct})$ method.

\section{MTT and colony formation assays}

3-(4,5-dimethylthiazol-2-yl)-2,5-diphenyltetrazolium bromide (MTT) assay was performed to detect cell growth. Cells were seeded into a 96 -well plate $\left(5 \times 10^{3}\right.$ cells/well $)$ and grew for 24,48 , and $72 \mathrm{~h}$. Cell viability was measured by MTT (Sigma-Aldrich Co.) assay as follows: $100 \mu \mathrm{L}$ MTT-medium mixed solution $(0.5 \mathrm{mg} / \mathrm{mL})$ was added to each well and incubated with cells for $4 \mathrm{~h}$. The formazan was dissolved in $200 \mu \mathrm{L}$ dimethyl sulfoxide following removal of the MTT solutions. Finally, the optical density was measured using a spectrophotometer at an absorption wavelength of $570 \mathrm{~nm}$.

For colony formation assay, the cells were seeded in to a six-well plate (400 cells/well), and cultured for 5 days. Then the clones were washed with $1 \times$ PBS and stained with crystal violet for $\sim 20 \mathrm{~min}$. Finally, the clones were imaged and quantified.

\section{Antibodies}

The antibodies purchased were as follows: anti-p53, anti-p21, and anti-p27 from Santa Cruz Biotechnology; anti- $\beta$-actin from Cell Signaling Technology; Alexa Fluor 488 goat anti-mouse IgG from Abcam. An anti-TSPAN12 antibody was generated by immunizing the rabbits with C-terminal peptides of TSPAN12 (EHTSMANSFNTHFEMEEL). The antibodies were affinity-purified against recombinant GST-TSPAN12 fusion protein coupled to Glutathione Sepharose 4B according to the manufacturer's instructions (GE Healthcare). The cells were lysed in a buffer containing $20 \mathrm{mM}$ Tris $\cdot \mathrm{HCl}$ (pH 7.5), $150 \mathrm{mM} \mathrm{NaCl}, 5 \mathrm{mM}$ EGTA, and $1 \%$ Nonidet P-40 with a mixture of protease inhibitors and phosphotase inhibitor (Sigma-Aldrich Co.) before immunoprecipitation and immunoblotting assays.

\section{Immunoblotting studies}

RIPA lysis buffer (Thermo Fisher Scientific) with protease inhibitor (Sigma-Aldrich Co.) was used to extract the total protein. The same amounts of total proteins were separated via sodium dodecyl sulfate-polyacrylamide gel electrophoresis gel and then transferred to polyvinylidene difluoride membranes (EMD Millipore). After blocking with 5\% milk, the membranes were incubated with the primary antibodies overnight at $4{ }^{\circ} \mathrm{C}$ and then with the horseradish peroxidaseconjugated secondary antibody (Santa Cruz Biotechnology). Signals were detected with enhanced chemiluminescence system (Bio-Rad).

\section{Immunofluorescence studies}

A549 and H1299 cells were cultured on glass slides in a 12-well plate and transfected with different plasmids. At $24 \mathrm{~h}$ post-transfection, the cells were fixed with $4 \%$ paraformaldehyde, permeabilized by $0.1 \%$ Triton X-100 in $1 \times$ PBS, and blocked in $3 \%$ bovine serum albumin in $1 \times$ PBS at $4^{\circ} \mathrm{C}$ overnight and then incubated with the antibody against p53, followed by incubation with secondary antibodies including Alexa Fluor 488 goat anti-mouse IgG. Finally, 4',6-diamidino-2-phenylindole was used to stain the nuclei for $5 \mathrm{~min}$ at room temperature. Fluorescence images were acquired with a Nikon microscope (E600).

\section{Cell apoptosis assays}

Four cell lines were constructed, including TSPAN12 knockdown A549 cells, TSPAN12 knockdown H1299 cells, and control cells for them, respectively. Cells were harvested, washed, and resuspended in the binding buffer containing Annexin V/fluorescein isothiocyanate (FITC) and propidium iodide (PI) (Beyotime). Cells were stained with Annexin V-FITC to monitor apoptosis cells and PI to detect dead cells, and then detected by flow cytometry (BD Biosciences, San Diego, CA, USA).

\section{Nude mice xenograft model}

All animals were maintained and used in accordance with the guidelines of the Institutional Animal Care and Use Committee of the Nanjing Medical University. All of the experimental procedures were approved by the Nanjing Medical University ethics commission. TSPAN12 knockdown H1299 cells and control cells in the logarithmic phase of growth were trypsinized, rinsed with $1 \times$ PBS for three times, and then resuspended in $100 \mu \mathrm{L} 1 \times$ PBS. Every six nude mice (6 weeks old, male) were inoculated subcutaneously with a clonal population of $5 \times 10^{6}$ cells in the upper right shoulders. Xenograft tumor sizes were measured by measuring two perpendicular diameters with digital calibers every 5 days after appearance of tumors and calculated according to the formula: $0.5 \times$ length $\times$ width $^{2}$. All of the mice were killed 1 month after inoculation and the tumors were removed instantly. 


\section{Statistical analyses}

Data were presented as the mean \pm SD from three independent experiments. Two-tailed Student's $t$-tests were used to evaluate the data. The difference was considered to be statistically significant at $p<0.05$ and $p<0.01$.

\section{Results}

\section{TSPANI 2 is upregulated in primary lung cancer tissues}

To detect the expression of TSPAN12 in lung cancer, we examined TSPAN12 expression in paracarcinoma lung tissue and lung cancer tissue by IHC. TSPAN12 was obviously upregulated in lung cancer tissues compared with normal tissues (Figure 1A). Most of the NSCLC tissues (68.8\%, $11 / 16$ cases) were found to exhibit high TSPAN12 expression. By contrast, most of the normal tissues $(76.7 \%, 23 / 30$ cases) expressed low TSPAN12 (Figure 1B). The qRT-PCR and Western blot analysis also indicated that mRNA and protein expression level of TSPAN12 was increased in NSCLC tissues, while the p53 level was lower in NSCLC tissues (Figure 1C and D). At the same time, TSPAN12 expression in 16 tumors and paired normal tissues from the patients was examined, and the results are presented in Table 1. Moreover, there was a negative correlation between the protein levels of TSPAN12 and p53 in NSCLC tissues $(r=-0.225, p<0.0001$; Figure 1E).

\section{TSPANI 2 is upregulated in human NSCLC cells}

To study the role of TSPAN12 in NSCLC, we used one normal human bronchial epithelial [16HBE] cells and three different NSCLC cell lines, H1299, A549, and A1650. We analyzed the TSPAN12 expression levels of these cell lines. We found that TSPAN12 mRNA levels were significantly increased in NSCLC cell lines compared with 16HBE cells (Figure 1F).
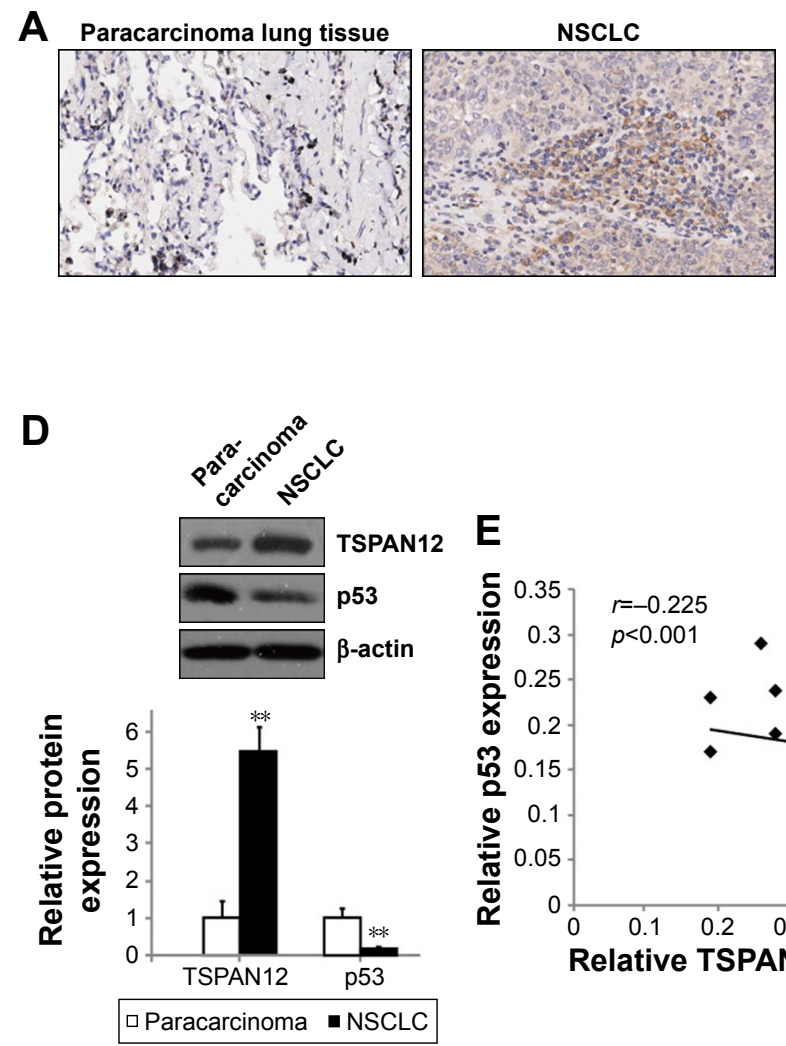

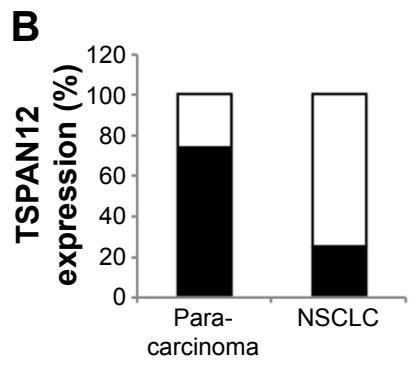

口 High - Low

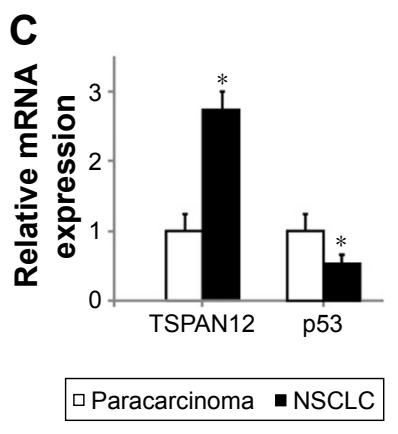

Figure I Expression of TSPANI 2 is increased in NSCLC cells and human NSCLC tissues.

Notes: (A) The expression of TSPANI 2 was detected in paracarcinoma lung tissue and lung cancer tissue by IHC, and representative samples are shown at $400 \times$ magnification. (B) The expression of TSPANI2 was quantified by $\mathrm{H}$-score in paracarcinoma lung tissue and lung cancer tissue. The samples were classified as low $(\mathrm{H}$-score $<200)$ or high (H-score $\geq 200$ ) TSPANI 2 expression. (C) The mRNA levels of TSPANI 2 in the paracarcinoma lung tissue and lung cancer tissue were detected by quantitative RT-PCR. (D) The protein levels of TSPANI 2 in the paracarcinoma lung tissue and lung cancer tissue were detected by Western blot. (E) Pearson correlation analysis of the protein expressions between TSPANI 2 and p53 in NSCLC tissues. (F) Quantitative RT-PCR analysis of TSPANI 2 mRNA levels in HBE cells and NSCLC cells (A549, HI299, and Al650). *p<0.05; **p $<0.01$.

Abbreviations: HBE, human bronchial epithelial; IHC, immunohistochemistry; NSCLC, non-small-cell lung carcinoma; RT-PCR, reverse transcriptase polymerase chain reaction; TSPAN I2, tetraspanin 12 . 
Table I Association between TSPANI 2 expression and clinicopathologic features of NSCLC patients

\begin{tabular}{|c|c|c|c|c|}
\hline \multirow{2}{*}{$\begin{array}{l}\text { Clinicopathological } \\
\text { parameter }\end{array}$} & \multirow{2}{*}{$\begin{array}{l}\text { No of } \\
\text { patients (16) }\end{array}$} & \multicolumn{3}{|c|}{ TSPAN I 2} \\
\hline & & $\begin{array}{l}\text { Lower } \\
(n=5)\end{array}$ & $\begin{array}{l}\text { Higher } \\
(n=I I)\end{array}$ & p-value \\
\hline Gender & & & & 0.0532 \\
\hline Male & 10 & 3 & 7 & \\
\hline Female & 6 & 2 & 4 & \\
\hline Age (years) & & & & 0.0483 \\
\hline$\leq 60$ & 12 & 4 & 8 & \\
\hline$>60$ & 4 & 1 & 3 & \\
\hline Size of tumor & & & & 0.0673 \\
\hline$\leq 3 \mathrm{~cm}$ & 9 & 3 & 6 & \\
\hline$>3 \mathrm{~cm}$ & 7 & 2 & 5 & \\
\hline Lymph node metastasis & & & & 0.0652 \\
\hline No & 5 & 2 & 3 & \\
\hline $\mathrm{NI}-3$ & 11 & 3 & 8 & \\
\hline Differentiation grade & & & & 0.235 \\
\hline Moderate-well & 10 & 3 & 7 & \\
\hline Poor & 6 & 2 & 4 & \\
\hline Histology type & & & & 0.035 \\
\hline Adenocarcinoma & 12 & 4 & 8 & \\
\hline Squamous carcinoma & 4 & I & 3 & \\
\hline Smoking & & & & 0.487 \\
\hline Yes & 9 & 2 & 7 & \\
\hline No & 7 & 3 & 4 & 0.002 \\
\hline \multicolumn{5}{|l|}{ TNM stage } \\
\hline $\mathrm{I} / \mathrm{II}$ & 13 & 4 & 9 & \\
\hline III & 3 & I & 2 & \\
\hline
\end{tabular}

Abbreviations: NSCLC, non-small-cell lung carcinoma; TNM, tumor-nodemetastasis; TSPANI2, tetraspanin 12 .

\section{Knockdown of TSPANI 2 inhibits cell proliferation and colony formation of NSCLC cell lines}

We also established A549 and H1299 cell lines with downregulated TSPAN12 expression by lentivirus infection. Western blot analysis and qRT-PCR analysis were conducted to determine the expression level of TSPAN12 in these cells (Figure 2A and B). The cell proliferation rate of these cells was investigated by MTT assay, and the results showed that the cell growth rate of NSCLC cells was significantly decreased by TSPAN12 downregulation (Figure 2C).

Next, colony formation was used to investigate the tumorigenesis potential of these cells. We found that the capacity of colony formation was reduced by TSPAN12 knockdown in NSCLC cells (Figure 2D). Therefore, these data suggest that TSPAN12 promotes the growth of NSCLC cells.

\section{TSPAN 2 regulates cell apoptosis in NSCLC cells}

To determine how TSPAN12 promotes cell proliferation and colony formation in NSCLC cells, we examined cell apoptosis in A549 and H1299 cells with TSPAN12 knockdown. Downregulation of TSPAN12 in NSCLC cells greatly promotes cell apoptosis (Figure 3A and B). Collectively, these results suggested that TSPAN12 regulates cell proliferation in NSCLC via cell apoptosis.

TSPAN I 2 silencing upregulates $\mathrm{p} 53, \mathrm{p} 2 \mathrm{I}$, and p27 in NSCLC cells

To determine whether p53 is a downstream target of TSPAN12 in NSCLC cells, expression of p53 protein in the cells with altered TSPAN12 expression was analyzed by immunofluorescence. The results showed that silencing of TSPAN12 expression in NSCLC cells dramatically increased p53 protein expression in A549 cells and wild-type p53 expression plasmid (pC53-SN3) in p53-null lung carcinoma H1299 cells. Furthermore, protein levels of p53 downstream genes like p21 and p27 were also changed after altering TSPAN12 expression in A549 and H1299 cells (Figure 3C and $\mathrm{D})$. These results indicate that TSPAN12 may regulate NSCLC cell proliferation and apoptosis through affecting p53 pathway.

\section{TSPAN 12 promotes the growth of NSCLC cells in vivo}

Next, we used a xenograft model to further elucidate the effect of TSPAN12 on tumor growth of NSCLC in vivo. H1299 cells with TSPAN12 knockdown were implanted into the nude mice. These mice were sacrificed in 1 month and tumors were removed. The growth of the NSCLC cells in these mice was measured by tumor volume and tumor weight. Consistent with our in vitro findings, tumors derived from TSPAN12 silenced H1299 cells grew at a much slower rate than those derived from control cells, as reflected in the significantly smaller tumor size, tumor volume (Figure 4A), and tumor weight (Figure 4B). Also, the mRNA level of TSPAN12 in these tumors was confirmed by qRT-PCR (Figure 4C). These observations, therefore, clearly indicated that TSPAN12 silencing can effectively suppress tumorigenicity of NSCLC cells in vivo.

\section{Discussion}

In this study, we have provided convincing evidence that TSPAN12 functions as a tumor promoter in human NSCLC. In the clinical NSCLC samples, TSPAN12 expression level was robustly upregulated as compared to the adjacent normal tissues. At the cellular level, knockdown of TSPAN12 in 
A

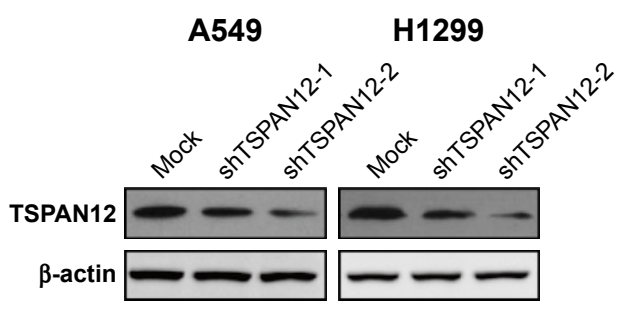

C

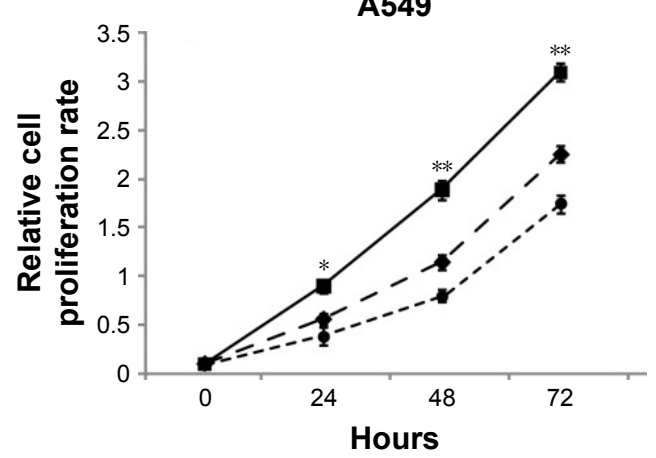

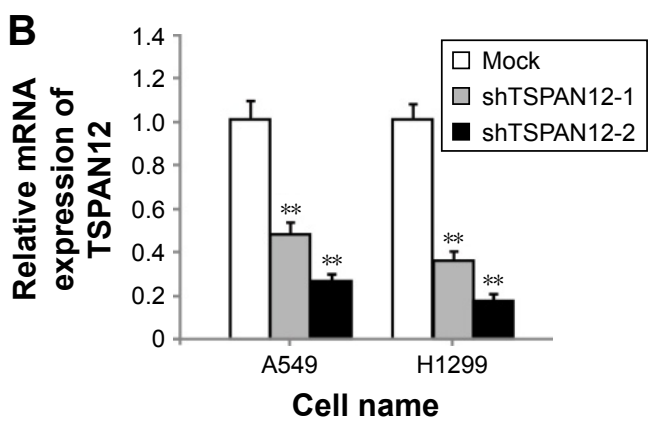

H1299

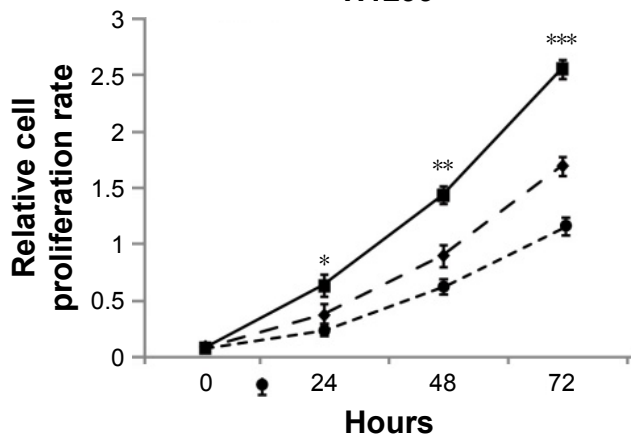

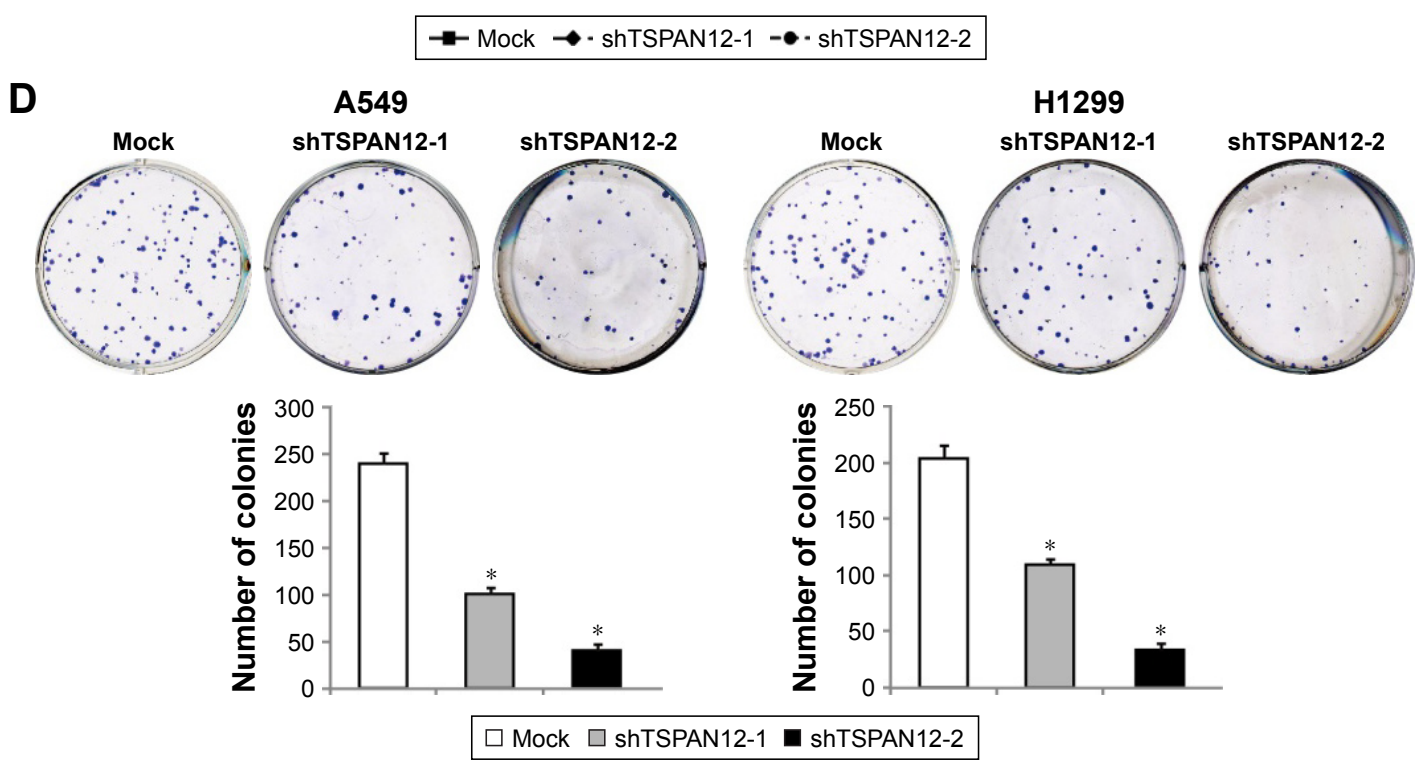

Figure 2 Silencing of TSPANI 2 inhibits NSCLC cell viability and proliferation.

Notes: (A) TSPANI2 protein expressions in A549 and HI299 cells with TSPANI2 shRNA or scrambled control shRNA (mock). (B) TSPANI2 mRNA level in A549 and HI299 cells with TSPANI 2 shRNA or scrambled control shRNA (mock). (C) CCK-8 assay was used to determine the cell proliferation rate of the cells at the time point as indicated. (D) Effect of TSPANI 2 downregulation on colony formation was measured in A549 and HI299 cells. The cells were seeded into six-well plates and cultured for 5 days, followed by crystal violet staining. The statistical analysis is shown in the histogram. These data are shown as mean \pm SD and $*$ for $p<0.05$, $* *$ for $p<0.01$ and $* * *$ for $p<0.001$

Abbreviations: NSCLC, non-small-cell lung carcinoma; shRNA, short hairpin ribonucleic acid; TSPANI2, tetraspanin I2; CCK, Cell Counting Kit.

A549 and H1299 cells significantly inhibited cell growth and colony formation. At the animal level, TSPAN12 silencing strongly inhibited the growth of NSCLC in nude mice. Mechanistically, TSPAN12 could modulate apoptosis and the expression of p21, p27, and p53 in NSCLC cells and such factors likely underlie its tumor-promoting activity in these cells.

p53 is one of the most important tumor suppressor genes and plays a major role in inhibition of angiogenesis. p53 can activate DNA repair proteins, initiate apoptosis, 

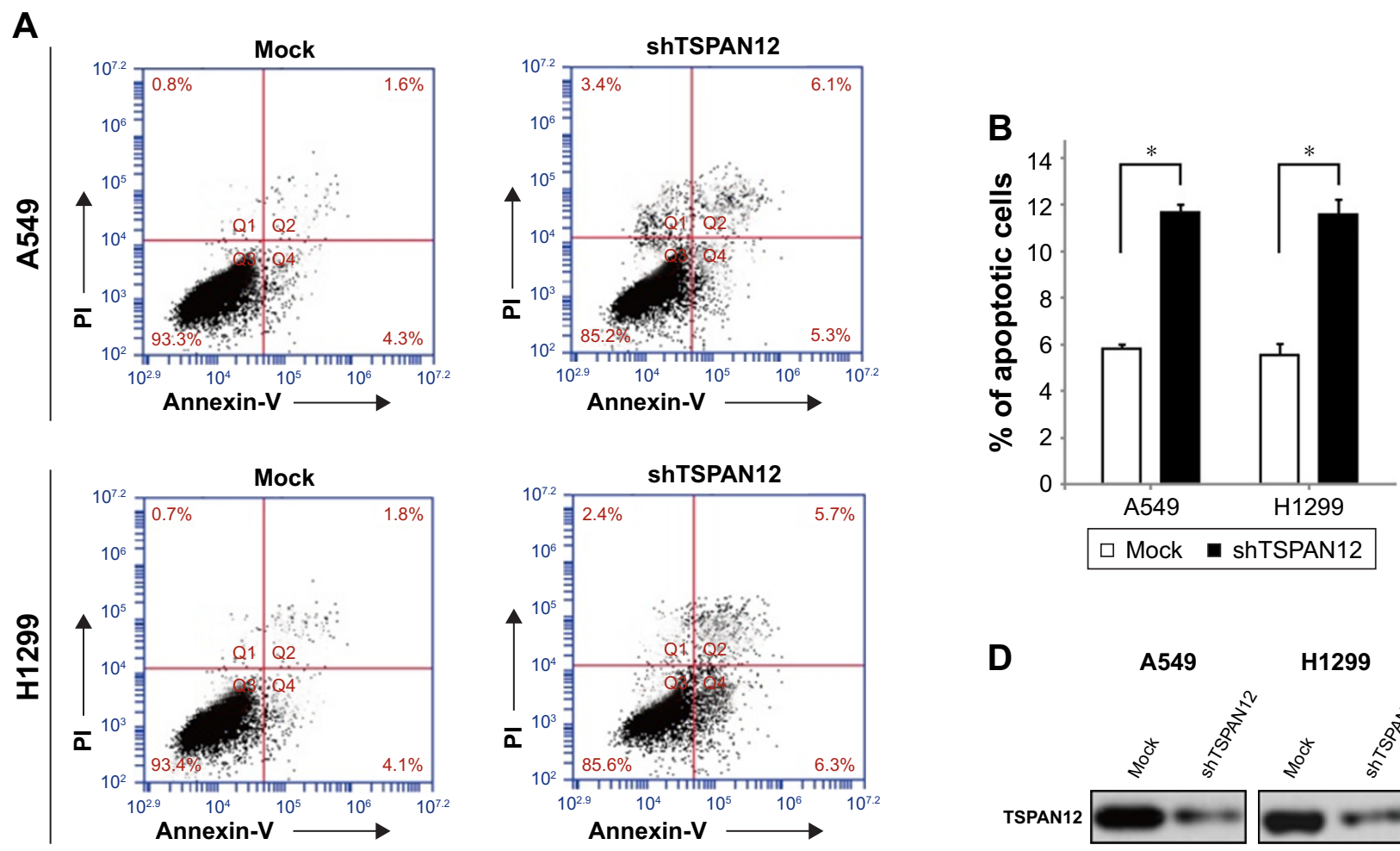

C

DAPI

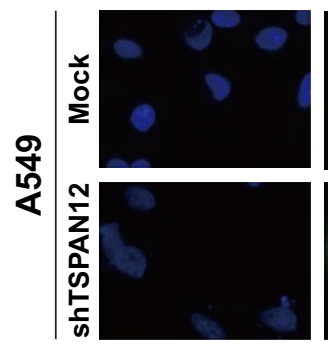

p53

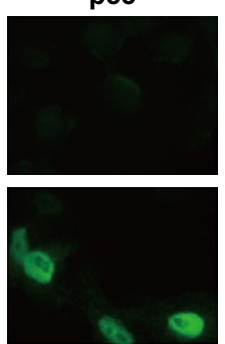

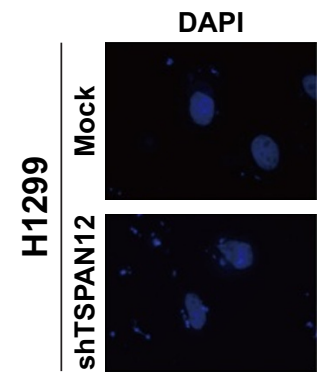

p53

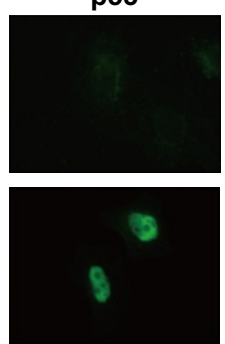

D

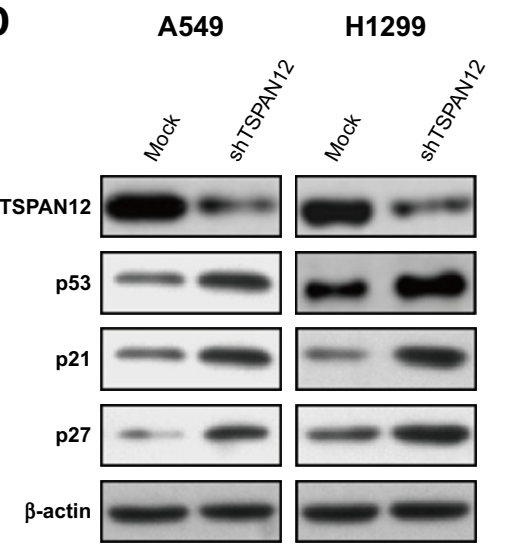

Figure 3 TSPANI 2 regulates $\mathrm{p} 53$ expression in NSCLC cells.

Notes: (A) Flow cytometry apoptosis assay of A549 and HI299 cells with Annexin V-fluorescein isothiocyanate and propidium iodide (PI) double staining ( $(\mathrm{n}=3)$. (B) The values of the Q2 plus Q4 quadrant represent apoptosis rates. Data are expressed as the mean \pm SD. ${ }^{*} p<0.05$. (C) Immunofluorescence stains were performed to assess the level of p53 in A549 and HI 299 cells with TSPANI 2 knockdown. (D) Western blot analysis was performed to assess the levels of p2I, p27, and p53 in A549 and HI299 cells with TSPANI2 knockdown.

Abbreviations: NSCLC, non-small-cell lung carcinoma; TSPANI2, tetraspanin 12.

or induce growth arrest by holding the cell cycle at the G1/S regulation point via transcriptional regulation. The activation of $\mathrm{p} 53$-mediated promotion of apoptosis in tumor cells is an important mechanism of antitumor drugs. Correlation analysis revealed a significantly inverse correlation between $\mathrm{p} 53$ and TSPAN12 protein levels in NSCLC specimens. To determine whether p53 is a downstream target of TSPAN12 in NSCLC, expression of $\mathrm{p} 53$ in the cells with altered TSPAN12 expression was evaluated by Western blot and immunofluorescence analysis. Furthermore, endogenous protein levels of p53 downstream genes like p21 and p27 were also changed after altering TSPAN12 expression in A549 and H1299 cells. However, molecular mechanisms underlying the link between TSPAN12 and p53 need further investigations.

Taken together, our study shows, for the first time, that TSPAN12 is overexpressed in human NSCLC tissues and NSCLC cell lines. Mechanistically, we have demonstrated that TSPAN12 silencing inhibits NSCLC cell proliferation and colony formation both in vitro and in vivo by affecting p53 pathway. High expression levels of TSPAN12 may serve as a novel molecular marker for human NSCLC and a promising target for drug development. 
A
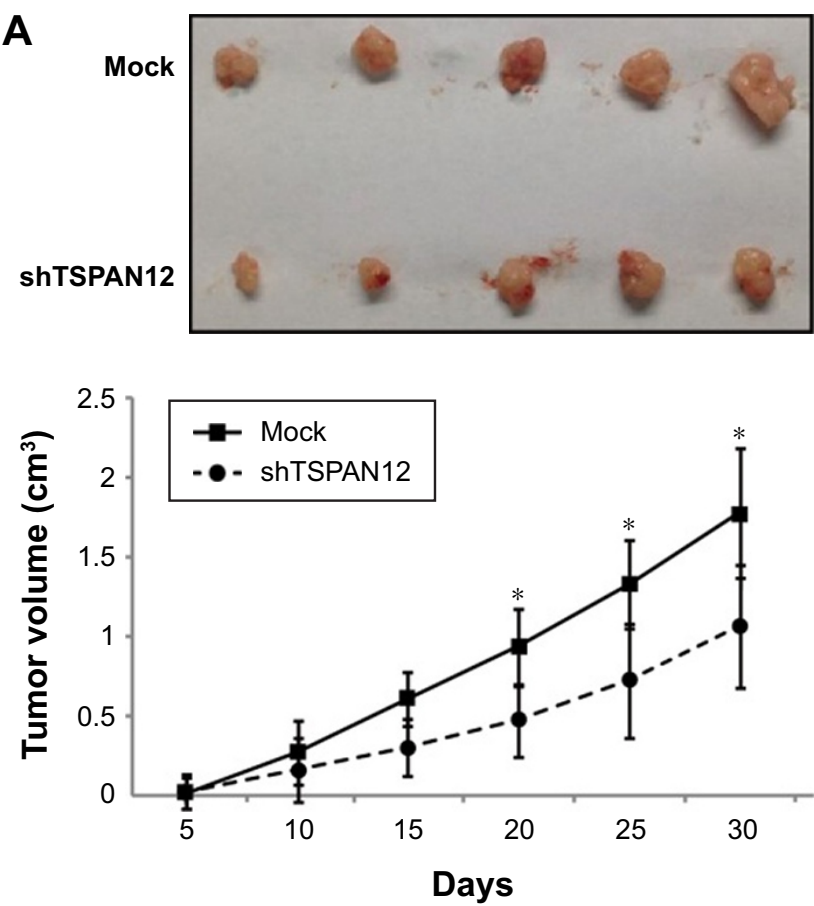

B

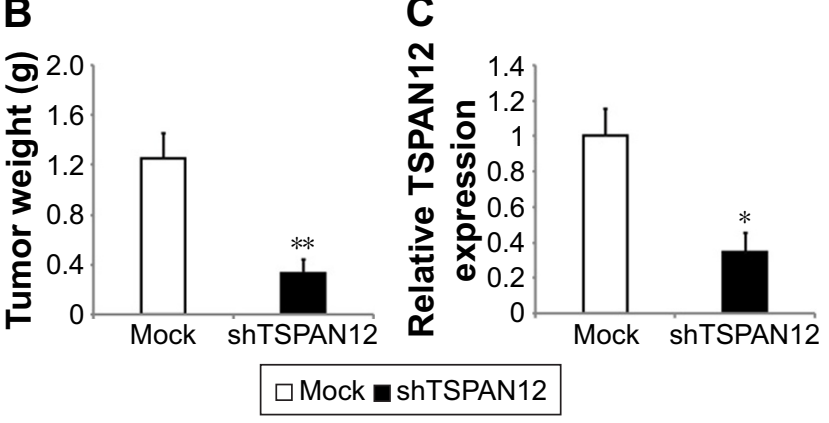

Figure 4 Downregulation of TSPANI 2 in lung carcinoma xenografts inhibits tumor growth in vivo.

Notes: (A) Tumor volume was calculated every 5 days after the injection of $\mathrm{HI} 299$ cells with TSPANI 2 knockdown. Tumor volume was calculated according to the formula $0.5 \times$ length $\times$ width $^{2}$. (B) The weight of the tumors. (C) Quantitative reverse transcriptase polymerase chain reaction analysis of TSPANI 2 mRNA levels in tumors. All the data are shown as mean $\pm S D$ and ${ }^{*} p<0.05$, ${ }^{* *} p<0.01$.

Abbreviation: TSPANI2, tetraspanin 12.

\section{Acknowledgment}

We declare that we have no financial and personal relationships with other people or organizations that can inappropriately influence our work. Zhongwu Hu and Daorong Hou share first authorship.

\section{Disclosure}

The authors report no conflicts of interest in this work.

\section{References}

1. Goldstraw P, Ball D, Jett JR, et al. Non-small-cell lung cancer. Lancet. 2011;378:1727-1740.

2. Ellis PG. Actionable biomarkers in a non-small cell lung cancer (NSCLC) clinical pathway (CP). Am Soc Clin Oncol. 2016;34:155.

3. Joerger M, Baty F, Früh M, et al. Circulating microRNA profiling in patients with advanced non-squamous NSCLC receiving bevacizumab/ erlotinib followed by platinum-based chemotherapy at progression (SAKK 19/05). Lung Cancer. 2014;85:306-313.

4. Joshi $\mathrm{P}$, Jeon $\mathrm{YJ}$, Laganà $\mathrm{A}$, et al. MicroRNA-148a reduces tumorigenesis and increases TRAIL-induced apoptosis in NSCLC. Proc Natl Acad Sci U S A. 2015;112:8650-8655.

5. Bean J, Brennan C, Shih JY, et al. MET amplification occurs with or without T790M mutations in EGFR mutant lung tumors with acquired resistance to gefitinib or erlotinib. Proc Natl Acad Sci U S A. 2007;104: 20932-20937.

6. Balak MN, Gong Y, Riely GJ, et al. Novel D761Y and common secondary T790M mutations in epidermal growth factor receptor-mutant lung adenocarcinomas with acquired resistance to kinase inhibitors. Clin Cancer Res. 2006;12:6494-6501.

7. Laskin JJ, Sandler AB. State of the art in therapy for non-small cell lung cancer. Cancer Invest. 2005;23:427-442.

8. Serru V, Dessen P, Boucheix C, Rubinstein E. Sequence and expression of seven new tetraspans. Biochim Biophys Acta. 2000;16:159-163.

9. Zoller M. Tetraspanins: push and pull in suppressing and promoting metastasis. Nat Rev Cancer. 2009;9:40-55.

10. Sala-Valdes M, Ailane N, Greco C, Rubinstein E, Boucheix C. Targeting tetraspanins in cancer. Expert Opin Ther Targets. 2012;16:985-997.

11. Hemler ME. Tetraspanin functions and associated microdomains. Nat Rev Mol Cell Biol. 2005;6:801-811.

12. Yanez-Mo M, Barreiro O, Gordon-Alonso M, Sala-Valdes M, SanchezMadrid F. Tetraspanin-enriched microdomains: a functional unit in cell plasma membranes. Trends Cell Biol. 2009;19:434-446.

13. Poulter JA, Ali M, Gilmour DF, et al. Mutations in TSPAN12 cause autosomal-dominant familial exudative vitreoretinopathy. Am J Hum Genet. 2010;86:248-253.

14. Junge HJ, Yang S, Burton JB, et al. TSPAN12 regulates retinal vascular development by promoting Norrin- but not Wnt-induced FZD4/betacatenin signaling. Cell. 2009;139:299-311.

15. Xu D, Sharma C, Hemler ME. Tetraspanin12 regulates ADAM10dependent cleavage of amyloid precursor protein. Faseb J. 2009;23: 3674-3681.

16. Knoblich K, Wang HX, Sharma C, et al. Tetraspanin TSPAN12 regulates tumor growth and metastasis and inhibits beta-catenin degradation. Cell Mol Life Sci. 2014;71:1305-1314.

17. Otomo R, Otsubo C, Matsushima-Hibiya Y, et al. TSPAN12 is a critical factor for cancer-fibroblast cell contact-mediated cancer invasion. Proc Natl Acad Sci U S A. 2014;111:18691-18696.

18. Vousden KH, Prives C. Blinded by the light: the growing complexity of p53. Cell. 2009;137:413-431.

19. Menendez D, Inga A, Resnick MA. The expanding universe of $\mathrm{p} 53$ targets. Nat Rev Cancer. 2009;9:724-737.

20. Stegh AH. Targeting the 53 signaling pathway in cancer therapy - the promises, challenges and perils. Expert Opin Ther Targets. 2012;16: $67-83$. 
OncoTargets and Therapy

\section{Publish your work in this journal}

OncoTargets and Therapy is an international, peer-reviewed, open access journal focusing on the pathological basis of all cancers, potential targets for therapy and treatment protocols employed to improve the management of cancer patients. The journal also focuses on the impact of management programs and new therapeutic agents and protocols on

patient perspectives such as quality of life, adherence and satisfaction The manuscript management system is completely online and includes a very quick and fair peer-review system, which is all easy to use. Visit http://www.dovepress.com/testimonials.php to read real quotes from published authors.

Submit your manuscript here: http://www.dovepress.com/oncotargets-and-therapy-journal 